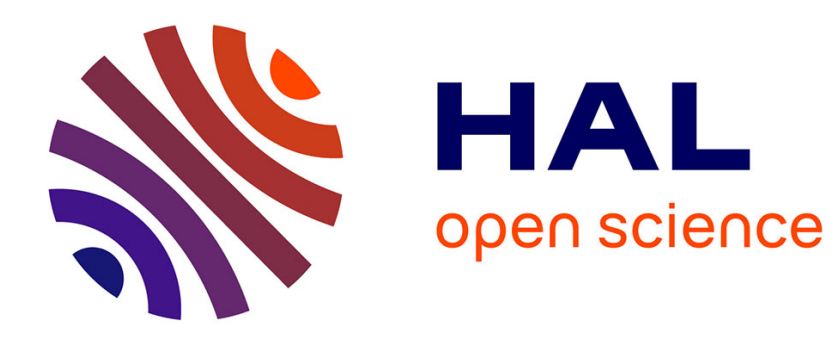

\title{
A Distributed Solution to the Network Reconstruction Problem
}

\author{
Fabio Morbidi, Alain Kibangou
}

\section{To cite this version:}

Fabio Morbidi, Alain Kibangou. A Distributed Solution to the Network Reconstruction Problem. Systems and Control Letters, 2014, 70, pp.85-91. 10.1016/j.sysconle.2014.05.008 . hal-01001828

\section{HAL Id: hal-01001828 \\ https://hal.science/hal-01001828}

Submitted on 5 Jun 2014

HAL is a multi-disciplinary open access archive for the deposit and dissemination of scientific research documents, whether they are published or not. The documents may come from teaching and research institutions in France or abroad, or from public or private research centers.
L'archive ouverte pluridisciplinaire HAL, est destinée au dépôt et à la diffusion de documents scientifiques de niveau recherche, publiés ou non, émanant des établissements d'enseignement et de recherche français ou étrangers, des laboratoires publics ou privés. 


\title{
A Distributed Solution to the Network Reconstruction Problem
}

\author{
Fabio Morbidi`a , Alain Y. Kibangou ${ }^{\mathrm{b}}$ \\ ${ }^{a}$ Inria, NeCS team, 655 Avenue de l'Europe, 38334 Montbonnot Saint Martin, France. \\ ${ }^{\mathrm{b}}$ Université Grenoble Alpes, CNRS, Inria, Gipsa-Lab, F-38000 Grenoble, France.
}

\begin{abstract}
It has been recently shown in [1] that by collecting noise-contaminated time series generated by a coupled-oscillator system at each node of a network, it is possible to robustly reconstruct its topology, i.e. determine the graph Laplacian. Restricting ourselves to linear consensus dynamics over undirected communication networks, in this paper we introduce a new dynamic average consensus least-squares algorithm to locally estimate these time series at each node, thus making the reconstruction process fully distributed and more easily applicable in the real world. We also propose a novel efficient method for separating the off-diagonal entries of the reconstructed Laplacian, and examine several concepts related to the trace of the dynamic correlation matrix of the coupled single integrators, which is a distinctive element of our network reconstruction method. The theory is illustrated with examples from computer, power and transportation systems.
\end{abstract}

Key words: Network reconstruction; Consensus algorithms; Distributed estimation; Networked systems

\section{Introduction}

In graph theory the "basic inverse problem" consists in determining the topology of a graph from its adjacency spectrum [2]. It is well known that: $i$ ) graphs with few distinct eigenvalues tend to have some kind of regularity; ii) cospectral graphs have the same number of closed walks of a given length; iii) there exist cospectral graphs which are not isomorphic. Therefore, the adjacency (and analogously the Laplacian) spectrum of a graph is not sufficient, in general, to unambiguously identify the graph topology.

Recently, the interest in the basic inverse problem has been revitalized by several works in the control and mathematical physics literatures, dealing with the reconstruction of the topology of a network of dynamical systems (this is also sometimes referred to as network "identification" or "exploration" problem). Uncovering the relationship between dynamics and network structure has indeed relevant applications in biology (biochemical, neural and ecological networks), finance, computer science (Internet and World Wide Web), transportation (delivery and distribution networks), and electrical engineering (power grids).

In [3], the interaction geometry among a known number of agents adopting (weighted) consensus-type algorithms for their coordination, is reconstructed using a grounding procedure inspired by experimental biology,

\footnotetext{
^ Corresponding author, Tel. : +33 (0)4 76615322

Email addresses: fabio.morbidi@inria.fr (Fabio Morbidi $^{\star}$ ), alain.kibangou@ujf-grenoble.fr (Alain Y. Kibangou).
}

called "node knockout" (see also [4]), while in [5] the consensus matrix is determined from its eigenstructure estimated in a distributed fashion.

Stochastic methods have lately emerged as powerful alternatives to the deterministic ones in $[3-5]$ : the main idea here is to reconstruct the network topology from noise-contaminated time series collected at each node of the graph. Noise is ubiquitous, e.g., in biological networks and relying on such a natural variability as a noninvasive network-identification tool appears extremely promising [6]. Several stochastic reconstruction methods have been proposed in the recent literature. In [7], the topology of a directed weighted network of LTI systems is estimated via power spectral analysis, while in [8] the authors formulate the problem of network reconstruction as a compressing sensing problem. Other approaches have exploited the sparsity of the network and used the Bayesian information criterion to measure the graph structure from stationary time series [9] or optimizationbased methods [10]: however, although the sparsity assumption is well justified in some applications (e.g. in biological networks), it may lead to poor results in other cases as shown in [11]. More related to the standard system-identification literature is the work in [6], where the authors formulated the network reconstruction problem as a variant of the spectral factorization problem, and [12] where the classical "direct method" of closedloop identification is utilized. However, in these works unknown noise sources are applied only to the states that are measured, which is an unrealistic assumption in many applications. Finally, in [1], an interesting connection between dynamic correlation and topology in noisy coupled-oscillator networks (where the noise magnitude 


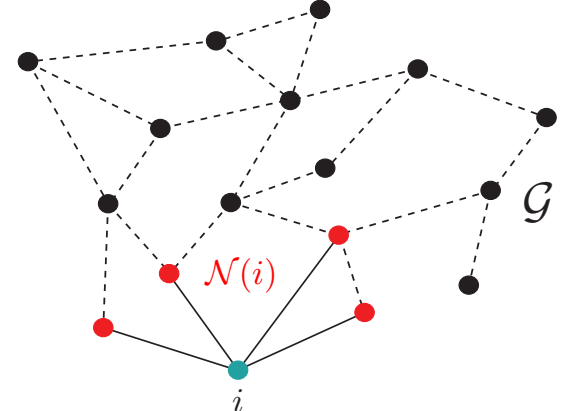

Fig. 1. Distributed network reconstruction: node $i$ must reconstruct the topology of the network $\mathcal{G}$ by only exchanging information with its set of neighbors $\mathcal{N}(i)$ (red nodes).

is known) has been unveiled. The reconstruction algorithm originally proposed in [1] has been recently improved in [13] by defining a different thresholding mechanism based on the largest eigenvalue of the Laplacian matrix estimated in a distributed fashion. However, all the aforementioned stochastic approaches are centralized or semi-centralized (as [13]), since they require the knowledge of time series at each node of the network, and thus impractical for real-world applications.

This paper builds upon [1] and extends it in several new directions. In particular, the original contributions of this work are threefold:

- The approach in [1] is made fully distributed by leveraging a new dynamic average consensus least-squares algorithm for the local estimation of the noisy time series at each node of the graph. In this way, each node is able to infer the topology of the overall undirected network (cf. Fig. 1): to the best of our knowledge, together with [5], this is the only completely-decentralized reconstruction algorithm available in the literature for networks of dynamical systems. An original stability analysis of the proposed dynamic average consensus least-squares estimator is also performed.

- A new robust and effective mechanism for separating the -1 and 0 off-diagonal entries of the reconstructed graph Laplacian is proposed. The method is based on $\kappa$-means clustering [14] and it is simpler than the thresholding criteria utilized in $[1,13]$.

- Interesting connections are shown between the trace of the dynamic correlation matrix of the coupled singleintegrator nodes (which plays a crucial role in our reconstruction mechanism), the total effective resistance of the network [15], and the $\mathrm{H}_{2}$ norm of noisy reduced consensus dynamics $[16,17]$.

Note that the distributed network-reconstruction algorithm proposed in this paper may represent a valid alternative (at least at small scales) to "web crawlers" for the World Wide Web and to "traceroute sampling" for the Internet, where there does not exist a vantage point with complete information about the overall structure of the system. Moreover, it may be useful for probing the structure of dynamically-changing networks, e.g. road networks, where links can appear/disappear over time because of accidents or works on the carriageway. The rest of this paper is organized as follows. Sect. 2 presents some background material. The main theoretical results of the work are introduced in Sect. 3 and Sect. 4, and the theory is illustrated with numerical simulations on realistic networks in Sect. 5. Finally, Sect. 6 summarizes the main contributions of the paper and outlines some promising future research directions.

\section{Preliminaries}

In this section we recall some notions of algebraic graph theory and robust control, and introduce the notation. Let $\mathcal{G}=(\mathcal{V}, \mathcal{E})$ be an undirected graph (or network), where $\mathcal{V}=\{1, \ldots, n\}$ is the set of nodes and $\mathcal{E}$ the set of links. $\mathcal{N}(i)$ will indicate the set of nodes adjacent to node $i$ in the graph $\mathcal{G}$. All graphs in this paper are finite, connected, with no self-loops and multiple links.

The adjacency matrix $\mathbf{A}=\left[a_{i j}\right]$ of graph $\mathcal{G}$ is an $n \times n$ matrix defined as

$$
a_{i j}= \begin{cases}1 & \text { if }\{i, j\} \in \mathcal{E} \\ 0 & \text { otherwise }\end{cases}
$$

The Laplacian matrix $\mathbf{L}=\left[\ell_{i j}\right]$ of graph $\mathcal{G}$ is an $n \times n$ symmetric positive semidefinite matrix defined as $\mathbf{L}=$ $\mathbf{D}-\mathbf{A}$ where $\mathbf{D}=\operatorname{diag}(\mathbf{A} \mathbb{1})$ is the degree matrix and $\mathbb{1}$ is a column vector of $n$ ones. From this definition, it follows that:

$$
\ell_{i j}= \begin{cases}\sum_{j=1}^{n} a_{i j} & \text { if } i=j \\ -1 & \text { if }\{i, j\} \in \mathcal{E}, \quad i, j \in\{1, \ldots, n\} \\ 0 & \text { otherwise. }\end{cases}
$$

Let $\mathbf{L}^{\dagger}$ be the (Moore-Penrose) pseudoinverse of the Laplacian matrix, $\mathbf{J}=\frac{1}{n} \mathbb{1}^{T}$ the $n \times n$ (rank one) averaging matrix, and $\mathbf{I}_{n}$ the $n \times n$ identity matrix. Then, we have that

$$
\mathbf{L}^{\dagger} \mathbf{L}=\mathbf{I}_{n}-\mathbf{J}
$$

which is the projection matrix onto the image of $\mathbf{L}$ [15]. From (1), it can be verified that:

$$
\mathbf{L}^{\dagger}=(\mathbf{L}+\mathbf{J})^{-1}-\mathbf{J}
$$

and that

$$
\operatorname{trace}\left(\mathbf{L}^{\dagger}\right)=\sum_{i=2}^{n} \frac{1}{\lambda_{i}(\mathbf{L})},
$$

where $0=\lambda_{1}(\mathbf{L})<\lambda_{2}(\mathbf{L}) \leq \ldots \leq \lambda_{n}(\mathbf{L})$ are the ordered eigenvalues of $\mathbf{L}$ [15]. Note that $\mathbf{L}^{\dagger}$ inherits from $\mathbf{L}$ the property of being symmetric and positive semidefinite. Moreover, $\mathbf{L}^{\dagger}$ and $\mathbf{L}$ share the same null space.

The $H_{2}$ norm of a general LTI system $\dot{\mathbf{x}}=\mathbf{A} \mathbf{x}+\mathbf{B} \mathbf{u}$, $\mathbf{y}=\mathbf{E} \mathbf{x}$, with A Hurwitz, is given by $\left(\operatorname{trace}\left(\mathbf{E X} \mathbf{E}^{T}\right)\right)^{1 / 2}$ where the positive semidefinite matrix $\mathbf{X}$ (the controllability Gramian) solves the algebraic Lyapunov equation $\mathbf{A X}+\mathbf{X} \mathbf{A}^{T}+\mathbf{B B}^{T}=\mathbf{0}$. In addition, if the pair $(\mathbf{A}, \mathbf{B})$ is controllable, $\mathbf{X}$ is positive definite.

Notation: $|\mathscr{S}|$ will denote the cardinality of the set $\mathscr{S}$, $\emptyset$ the empty set, $\operatorname{Re}(z)$ and $|z|$ the real part and modulus of the complex number $z$, respectively, $\otimes$ the Kronecker product, blkdiag $(\cdot)$ a block-diagonal matrix, $\mathbb{E}[\cdot]$ the expectation operator, $\|\cdot\|_{2}$ the Euclidean norm of a vector and $\|\cdot\|_{\mathcal{L}_{2}}$ the total energy or $\mathcal{L}_{2}$ norm of a vector-valued signal. 


\section{Problem formulation}

For the reader's convenience we briefly review here the main results in [1], which form the basis for our subsequent developments. We will start with a general formulation dealing with directed graphs, and then we will specialize our results to undirected networks with coupled single-integrator node dynamics.

Consider a directed network of $n$ nonidentical coupled oscillators where $\mathbf{x}_{i} \in \mathbb{R}^{m}, i \in\{1, \ldots, n\}$, denotes the state variable of the $i$-th oscillator and $\mathbf{x}_{i}(0)$ the initial state. In the presence of noise, the dynamics of the whole oscillator system can be expressed as

$$
\dot{\mathbf{x}}_{i}=\mathbf{F}_{i}\left(\mathbf{x}_{i}\right)-\gamma \sum_{j=1}^{n} \ell_{i j} \mathbf{H}\left(\mathbf{x}_{j}\right)+\boldsymbol{\eta}_{i},
$$

where $\mathbf{F}_{i}: \mathbb{R}^{m} \rightarrow \mathbb{R}^{m}$ is the intrinsic dynamics of the $i$-th oscillator, $\mathbf{H}: \mathbb{R}^{m} \rightarrow \mathbb{R}^{m}$ is the coupling function of the oscillators, $\gamma>0$ is the coupling strength, and $\boldsymbol{\eta}_{i} \in \mathbb{R}^{m}$ is the zero-mean white noise with covariance matrix $\mathbf{Q}_{i}$ associated with the $i$-th oscillator. We will assume that $\mathbf{x}_{i}(0)$ and $\boldsymbol{\eta}_{i}$ are uncorrelated for any $i \in$ $\{1, \ldots, n\}$, and that $\boldsymbol{\eta}_{i}$ and $\boldsymbol{\eta}_{j}$ are uncorrelated for any $i \neq j, i, j \in\{1, \ldots, n\}$. Note that (4) encompasses several well-known coupled-oscillator systems, such as $\mathrm{Ku}$ ramoto phase oscillators and Rössler dynamics. Let $\overline{\mathbf{x}}_{i}$ be the counterpart of $\mathbf{x}_{i}$ in the absence of noise and $\boldsymbol{\xi}_{i}$ a small perturbation. Then we can write $\mathbf{x}_{i}=\overline{\mathbf{x}}_{i}+\boldsymbol{\xi}_{i}$ and plugging it in (4), we obtain the variational equation

$$
\dot{\boldsymbol{\xi}}_{i}=J\left[\mathbf{F}_{i}\left(\overline{\mathbf{x}}_{i}\right)\right] \boldsymbol{\xi}_{i}-\gamma \sum_{j=1}^{n} \ell_{i j} J\left[\mathbf{H}\left(\overline{\mathbf{x}}_{j}\right)\right] \boldsymbol{\xi}_{j}+\boldsymbol{\eta}_{i}
$$

where $J\left[\mathbf{F}_{i}(\cdot)\right]$ and $J[\mathbf{H}(\cdot)]$ are the $m \times m$ Jacobian matrices of $\mathbf{F}_{i}$ and $\mathbf{H}$, respectively. Now, let $\boldsymbol{\xi}=\left[\boldsymbol{\xi}_{1}^{T}, \ldots, \boldsymbol{\xi}_{n}^{T}\right]^{T} \in \mathbb{R}^{m n}$ be the deviation vector, $\boldsymbol{\eta}=\left[\boldsymbol{\eta}_{1}^{T}, \ldots, \boldsymbol{\eta}_{n}^{T}\right]^{T} \in \mathbb{R}^{m n}$ the noise vector, and

$$
\mathbf{C} \triangleq \frac{1}{\mathrm{~T}} \int_{0}^{\mathrm{T}} \boldsymbol{\xi}(t) \boldsymbol{\xi}^{T}(t) d t
$$

the dynamic correlation matrix of the oscillators, where $[0, \mathrm{~T}]$ is the (long) time interval in which the time average is performed. We then have that:

$$
\begin{aligned}
\mathbf{0} & =\frac{d}{d t}\left[\frac{1}{\mathrm{~T}} \int_{0}^{\mathrm{T}} \boldsymbol{\xi}(t) \boldsymbol{\xi}^{T}(t) d t\right]=\mathbf{M} \mathbf{C}+\mathbf{C M}^{T} \\
& +\frac{1}{\mathrm{~T}} \int_{0}^{\mathrm{T}} \boldsymbol{\eta}(t) \boldsymbol{\xi}^{T}(t) d t+\frac{1}{\mathrm{~T}} \int_{0}^{\mathrm{T}} \boldsymbol{\xi}(t) \boldsymbol{\eta}^{T}(t) d t
\end{aligned}
$$

with $\mathbf{M}=J[\mathbf{F}(\overline{\mathbf{x}})]-\gamma \mathbf{L} \otimes J[\mathbf{H}(\overline{\mathbf{x}})]$ where $J[\mathbf{F}(\overline{\mathbf{x}})]=$ $\operatorname{blkdiag}\left(J\left[\mathbf{F}_{1}\left(\overline{\mathbf{x}}_{1}\right)\right], \ldots, J\left[\mathbf{F}_{n}\left(\overline{\mathbf{x}}_{n}\right)\right]\right)$ and $\overline{\mathbf{x}}=\left[\overline{\mathbf{x}}_{1}^{T}, \ldots, \overline{\mathbf{x}}_{n}^{T}\right]^{T}$ $\in \mathbb{R}^{m n}$. Assuming that $\mathbf{M}$ is Hurwitz, after few algebraic manipulations we can rewrite (6) as

$$
\mathbf{M} \mathbf{C}+\mathbf{C M}^{T}+2 \mathbf{Q}=\mathbf{0},
$$

where $\mathbf{Q}=\operatorname{blkdiag}\left(\mathbf{Q}_{1}, \ldots, \mathbf{Q}_{n}\right)$. Note that (7), an algebraic Lyapunov equation in the unknown $\mathbf{C}$, reveals a general relationship between the dynamic correlation matrix $\mathbf{C}$ and the Laplacian $\mathbf{L}$ in the presence of noise with covariance matrix $\mathbf{Q}$. It is well known that if $\mathbf{M}$ is Hurwitz and $\mathbf{Q}$ positive semidefinite, the unique solution of (7) is positive semidefinite and it has the representation:

$$
\mathbf{C}=2 \int_{0}^{\infty} e^{\mathbf{M} t} \mathbf{Q} e^{\mathbf{M}^{T} t} d t
$$

If we assume that $\mathbf{M}=\mathbf{M}^{T}$ and $\mathbf{Q}=\frac{\sigma^{2}}{2} \mathbf{I}_{m n}, \sigma>0$, the integral in (8) can be explicitly computed and we obtain $\mathbf{C}=\frac{\sigma^{2}}{2} \mathbf{M}^{-1}$. If we further assume a linear coupling between the oscillators, i.e. $J[\mathbf{H}(\cdot)]=\mathbf{I}_{m n}$, no intrinsic dynamics, i.e. $\mathbf{F}_{i}(\cdot)=\mathbf{0}$ for all $i$, and one-dimensional state variables, i.e. $m=1$, we find the following simple formula relating the dynamic correlation matrix $\mathbf{C}$ to the Laplacian $\mathbf{L}$ :

$$
\mathbf{C}=\frac{\sigma^{2}}{2 \gamma} \mathbf{L}^{\dagger}
$$

Note that this corresponds to the case of nodes with coupled single-integrator dynamics driven by white noise, communicating over an undirected graph $\mathcal{G}$, i.e. to the noisy consensus dynamics

$$
\dot{\mathbf{x}}=-\gamma \mathbf{L} \mathbf{x}+\boldsymbol{\eta}
$$

where $\mathbf{x}=\left[x_{1}, \ldots, x_{n}\right]^{T} \in \mathbb{R}^{n}$ and $\boldsymbol{\eta} \in \mathbb{R}^{n}$. This simplified yet meaningful scenario will be considered in the rest of this paper. Henceforth, we also assume that the number $n$ of nodes of $\mathcal{G}$ is known. Note that from (9), we obtain

$$
\mathbf{L}=\frac{\sigma^{2}}{2 \gamma} \mathbf{C}^{\dagger}=\left[\frac{2 \gamma}{\sigma^{2}} \mathbf{C}+\mathbf{J}\right]^{-1}-\mathbf{J}
$$

where the second equality follows from (2). We then see that the entire network structure, embodied in the Laplacian matrix $\mathbf{L}$, can be extracted from the knowledge of noise-corrupted time series. In fact, recalling the definition of $\boldsymbol{\xi}$, the dynamic correlation matrix can be computed as

$$
\mathbf{C}=\frac{1}{\mathrm{~T}} \int_{0}^{\mathrm{T}}(\mathbf{x}(t)-\bar{x}(t) \mathbb{1})(\mathbf{x}(t)-\bar{x}(t) \mathbb{1})^{T} d t,
$$

where

$$
\bar{x}(t)=\frac{1}{n} \sum_{i=1}^{n} x_{i}(t)=\frac{1}{n} \mathbb{1}^{T} \mathbf{x}(t) .
$$

Since $\mathbf{x}(t)-\bar{x}(t) \mathbb{1}=\left(\mathbf{I}_{n}-\mathbf{J}\right) \mathbf{x}(t)$, we can rewrite equation (11) as

$$
\mathbf{L}=\left[\frac{2 \gamma}{\sigma^{2}}\left(\mathbf{I}_{n}-\mathbf{J}\right) \mathbf{C}_{\mathrm{R}}\left(\mathbf{I}_{n}-\mathbf{J}\right)+\mathbf{J}\right]^{-1}-\mathbf{J}
$$

where

$$
\mathbf{C}_{\mathrm{R}}=\frac{1}{\mathrm{~T}} \int_{0}^{\mathrm{T}} \mathbf{x}(t) \mathbf{x}^{T}(t) d t
$$

Since the integral in (13) cannot be analytically computed in practice, assuming $\mathrm{S}$ discrete observations in 
the time interval $[0, T]$, we can approximate $\mathbf{C}_{R}$ as

$$
\mathbf{C}_{\mathrm{R}} \simeq \frac{1}{\mathrm{~S}} \sum_{k=0}^{\mathrm{S}-1} \mathbf{x}(k) \mathbf{x}^{T}(k)
$$

Note that in order to compute the right-hand side of equation (14), we need to know the time series $\left\{x_{i}(0), x_{i}(1), \ldots, x_{i}(\mathrm{~S}-1)\right\}$ at each node of the graph. This means that in its present form, the network reconstruction algorithm relies on global information and it is thus centralized.

Next, we will introduce a practical method to separate the off-diagonal entries of the reconstructed Laplacian (Sect. 3.1), and highlight parallels between the trace of the dynamic correlation matrix $\mathbf{C}$ and concepts from other fields (Sect. 3.2). In Sect. 4, we will finally present a consensus-based least-squares estimation algorithm in order to make the reconstruction process fully distributed and computationally treatable: in this way, by only interacting with its neighbors, each node will be able to locally and efficiently estimate the topology of the overall network.

\subsection{Separation of the off-diagonal entries of the recon- structed Laplacian}

Fig. 2 shows the distribution of the off-diagonal entries of matrix $\frac{\sigma^{2}}{2 \gamma} \mathbf{C}^{\dagger}$ for a social network with 62 nodes and 159 links [18], obtained by simulating system (10) with $\gamma=1$ and $\sigma^{2}=2$ for 2000 seconds from a random initial condition. The distribution has one peak centered at -1 , corresponding to the existent links in the network and the other centered at 0 , corresponding to the zero elements of $\mathbf{L}$. Note that if $\mathbf{L}$ were perfectly reconstructed from $\frac{\sigma^{2}}{2 \gamma} \mathbf{C}^{\dagger}$, the two peaks would be sharp vertical lines. Since this does not occur in practice, a threshold needs to be determined to separate the two peaks

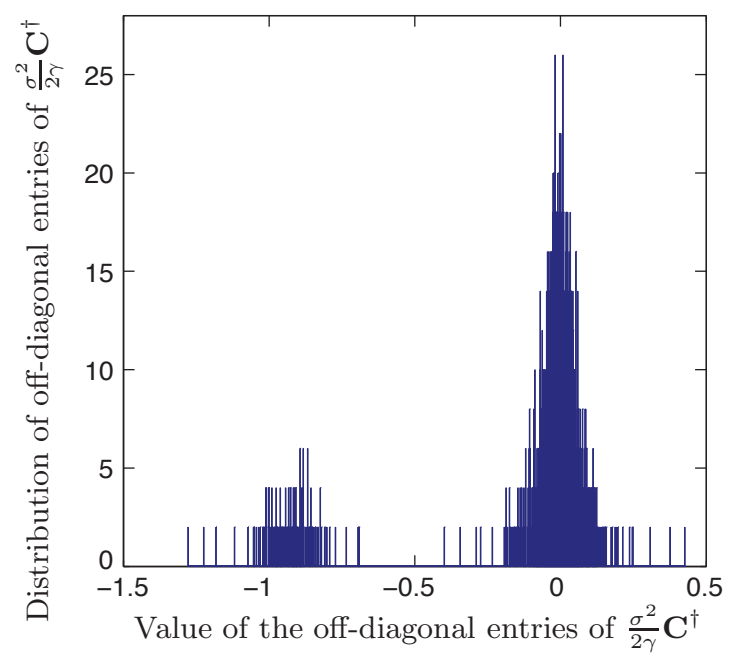

Fig. 2. Distribution of the off-diagonal entries of matrix $\frac{\sigma^{2}}{2 \gamma} \mathbf{C}^{\dagger}$ for a network with 62 nodes [18]. We set $\gamma=1, \sigma^{2}=2$ and run (10) for 2000 seconds.

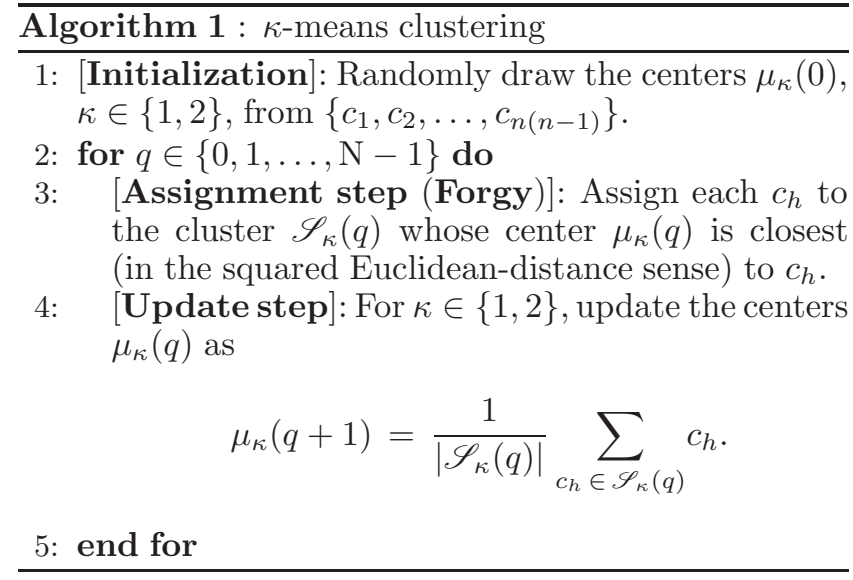

and distinguish the existent from the nonexistent links. An analytical procedure was proposed in [1] for finding such a threshold: however, it is neither immediate, nor appears well-physically motivated. On the other hand, the thresholding mechanism in [13], which is based on the largest eigenvalue of the graph Laplacian, is not described in sufficient detail so that it can be replicated herein.

As a simple yet effective alternative to the thresholding methods in $[1,13]$, in this paper we propose to use $\kappa$-means clustering [14]. Let $\mathbf{c}=\left[c_{h}\right] \in \mathbb{R}^{n(n-1)}$ be the vector of off-diagonal entries of $\frac{\sigma^{2}}{2 \gamma} \mathbf{C}^{\dagger}$ listed in the natural order. In our case, the clustering problem amounts to partition the entries of $\mathbf{c}$ into two clusters $\mathscr{S}_{1}, \mathscr{S}_{2}$ where $\mathscr{S}_{1}$ is relative to the -1 values and $\mathscr{S}_{2}$ to the 0 values $\left(\mathscr{S}_{1} \cap \mathscr{S}_{2}=\emptyset, \mathscr{S}_{1} \cup \mathscr{S}_{2}=\left\{c_{1}, c_{2}, \ldots, c_{n(n-1)}\right\}\right)$, so as to minimize the squared distortion:

$$
\min _{\mathscr{S}_{1}, \mathscr{S}_{2}, \mu_{1}, \mu_{2}} \sum_{\kappa \in\{1,2\}} \sum_{c_{h} \in \mathscr{S}_{\kappa}} \frac{1}{2}\left|c_{h}-\mu_{\kappa}\right|^{2},
$$

where $\mu_{\kappa}$ is the center of cluster $\mathscr{S}_{\kappa}, \kappa \in\{1,2\}$. This combinatorial optimization problem is nonconvex and exhaustive search for the optimal clusters is intractable. This intractability motivates low-complexity heuristics such as the $\kappa$-means algorithm. The algorithm uses an iterative refinement technique: in fact, it alternates between minimizing the squared distortion over $\left\{\mathscr{S}_{1}, \mathscr{S}_{2}\right\}$ (assignment step) and $\left\{\mu_{1}, \mu_{2}\right\}$ (update step), see Algorithm 1. The algorithm has converged when the assignments no longer change. Since both the steps optimize the squared distortion and there only exists a finite number of assignments, the algorithm must converge to a (local) minimum after $\mathrm{N}<\infty$ steps. Once convergence has been achieved, the Laplacian matrix can be recovered, for $h \in\{1, \ldots, n(n-1)\}, i \neq j$, $i, j \in\{1, \ldots, n\}$, as

$$
\widehat{\ell}_{i j}=\left\{\begin{array}{lll}
-1 & \text { if } \quad & c_{h} \in \mathscr{S}_{1}(\mathrm{~N}-1), \\
0 & \text { if } \quad & c_{h} \in \mathscr{S}_{2}(\mathrm{~N}-1)
\end{array}\right.
$$

and $\widehat{\ell}_{i i}=\sum_{j=1, j \neq i}^{n}\left|\widehat{\ell}_{i j}\right|, i \in\{1, \ldots, n\}$. 


\subsection{Physical interpretations of the correlation matrix}

\subsubsection{Effective graph resistance}

Assume the graph $\mathcal{G}$ is seen as an electrical circuit, where a link corresponds to a unit resistor. The effective resistance between a pair of nodes $i$ and $j$, is the electrical resistance measured across nodes $i$ and $j$. The total effective resistance, $R_{\mathrm{tot}}$, is defined as the sum of the effective resistance between all distinct pairs of nodes of $\mathcal{G}$ [15]. It has been shown (see, e.g., [15, Sect. 2.5]) that $R_{\text {tot }}$ depends on the spectrum of the graph Laplacian as

$$
R_{\mathrm{tot}}=n \sum_{i=2}^{n} \frac{1}{\lambda_{i}(\mathbf{L})}
$$

Hence, by recalling (3), we obtain the following concise formula relating the total effective resistance and the dynamic correlation matrix $\mathbf{C}$

$$
R_{\mathrm{tot}}=\frac{2 \gamma n}{\sigma^{2}} \operatorname{trace}(\mathbf{C})
$$

\subsubsection{Consensus robustness and the $\mathrm{H}_{2}$ norm}

Since system (10) is only marginally stable in the noisefree case, let us restrict to the dynamics on $\operatorname{span}(\mathbb{1})^{\perp}$, i.e. on the subspace of $\mathbb{R}^{n}$ orthogonal to the subspace spanned by $\mathbb{1}$. Let the matrix $\Theta \in \mathbb{R}^{(n-1) \times n}$ be such that $\boldsymbol{\Theta} \mathbb{1}=\mathbf{0}, \boldsymbol{\Theta} \boldsymbol{\Theta}^{T}=\mathbf{I}_{n-1}$ and define $\mathbf{r} \triangleq \boldsymbol{\Theta} \mathbf{x}$. By differentiating $\mathbf{r}$ with respect to time, we obtain:

$$
\dot{\mathbf{r}}=-\gamma \mathbf{L}^{*} \mathbf{r}+\boldsymbol{\Theta} \boldsymbol{\eta},
$$

where $\mathbf{L}^{*}=\boldsymbol{\Theta} \mathbf{L} \Theta^{T}$ is the reduced Laplacian matrix (note that $\mathbf{L}^{*}$ has the same eigenvalues of $\mathbf{L}$ except for the zero eigenvalue). Let us assume that the output equation of system (15) is

$$
\mathbf{y}=\mathbf{I}_{n-1} \mathbf{r},
$$

then we get the following result (for the proof the reader is referred to [17, Lemma 2]).

Proposition 1 Let the undirected graph $\mathcal{G}$ be connected and $\sigma^{2}=1$. The $H_{2}$ norm of system (15)-(16) is

$$
\mathcal{H}=\left(\sum_{i=2}^{n} \frac{1}{2 \gamma \lambda_{i}(\mathbf{L})}\right)^{1 / 2}=(\operatorname{trace}(\mathbf{C}))^{1 / 2}
$$

Let us now define $\boldsymbol{\Sigma}(t) \triangleq \mathbb{E}\left[\mathbf{r}(t) \mathbf{r}^{T}(t)\right]$. Note that $\mathcal{H}$ in Prop. 1 coincides with the measure of robustness to white-noise inputs of system (15) considered in [17]

$$
\mathcal{H} \triangleq \lim _{t \rightarrow \infty} \mathbb{E}\left[\|\mathbf{r}(t)\|_{2}\right]=\lim _{t \rightarrow \infty}(\operatorname{trace}(\boldsymbol{\Sigma}(t)))^{1 / 2} .
$$

This definition of robustness corresponds to the steadystate least-mean-square deviation introduced in [16] for discrete-time consensus dynamics: a related $\mathrm{H}_{2}$ normbased notion quantifying the deviation from consensus, is called (first-order) network coherence in [19].

Finally, under the same assumptions of Prop. 1, we have the following geometric interpretation of matrix $\mathbf{C}$ in relation to the properties of the controllability Gramian $\left(2 \gamma \mathbf{L}^{*}\right)^{-1}$ of system (15), cf. [20, Th. 6.18].
Proposition 2 (Reachable set with bounded noise) The closure of the set of all the states of system (15) reachable from $\mathbf{r}(0)=\mathbf{0}$ with noise bounded as

$$
\|\boldsymbol{\eta}\|_{\mathcal{L}_{2}}^{2} \triangleq \int_{0}^{\infty} \boldsymbol{\eta}^{T}(t) \boldsymbol{\eta}(t) d t \leq 1
$$

is given by the ellipsoid

$$
\mathscr{T}=\left\{\mathbf{r}: \mathbf{p}^{T} \mathbf{r} \leq\left(\mathbf{p}^{T} \boldsymbol{\Theta} \mathbf{C} \boldsymbol{\Theta}^{T} \mathbf{p}\right)^{1 / 2}, \forall \mathbf{p} \in \mathbb{R}^{n-1}\right\} .
$$

In other words, Prop. 2 says that the points in the state space of system (15) that can be reached using an excitation with total energy one, are given by an ellipsoid determined by $\mathbf{C}$.

\section{Distributed network-state estimation}

As aforementioned, in this section we will present a consensus-based strategy in order to make the reconstruction procedure described in Sect. 3 fully distributed. To this end, let us introduce the following measurement equation for node $i$ in the network $\mathcal{G}$ :

$$
\mathbf{y}_{i}(k)=\mathbf{E}_{i} \mathbf{x}(k), \quad k \in\{0,1, \ldots, \mathrm{S}-1\},
$$

where $\mathbf{E}_{i} \in\{0,1\}^{(|\mathcal{N}(i)|+1) \times n}$ is a selection matrix (the number of observations $\mathrm{S}$ is assumed to be known a priori by each node). In other words, at time instant $k \in\{0,1, \ldots, \mathrm{S}-1\}$, node $i$ measures its own state $x_{i}(k)$ (the first component of vector $\mathbf{y}_{i}(k)$ ), and the state $x_{j}(k)$ of $j \in \mathcal{N}(i)$. By collecting the measurements of all the agents at time $k$, we obtain $\mathbf{y}(k)=\mathbf{E} \mathbf{x}(k)$, $k \in\{0,1, \ldots, \mathbf{S}-1\}$, where $\mathbf{y}=\left[\mathbf{y}_{1}^{T}, \ldots, \mathbf{y}_{n}^{T}\right]^{T}$ and $\mathbf{E}=\left[\mathbf{E}_{1}^{T}, \ldots, \mathbf{E}_{n}^{T}\right]^{T}$. If the columns of $\mathbf{E}$ are linearly independent (i.e. $\mathbf{E}^{T} \mathbf{E}$ is invertible), then the leastsquares estimate of $\mathbf{x}(k)$ is

$$
\begin{aligned}
\widehat{\mathbf{x}}(k) & =\mathbf{E}^{\dagger} \mathbf{y}(k)=\left(\mathbf{E}^{T} \mathbf{E}\right)^{-1} \mathbf{E}^{T} \mathbf{y}(k) \\
& =\left[\frac{1}{n} \sum_{i=1}^{n} \mathbf{E}_{i}^{T} \mathbf{E}_{i}\right]^{-1}\left[\frac{1}{n} \sum_{i=1}^{n} \mathbf{E}_{i}^{T} \mathbf{y}_{i}(k)\right] .
\end{aligned}
$$

Note that the two quantities inside the square brackets in (17), can be computed in a distributed fashion using consensus-based algorithms. Let us then assume that node $i$ maintains a matrix $\mathbf{P}_{i} \in \mathbb{R}^{n \times n}$ and a vector $\mathbf{z}_{i} \in \mathbb{R}^{n}$, and executes the following iterations for $h \in$ $\{0,1, \ldots, D-1\}, D>1$,

$$
\begin{aligned}
& \mathbf{P}_{i}(h+1)=\mathbf{P}_{i}(h)+\varsigma \sum_{j \in \mathcal{N}(i)}\left(\mathbf{P}_{j}(h)-\mathbf{P}_{i}(h)\right), \\
& \mathbf{z}_{i}(h+1)=\mathbf{z}_{i}(h)+\varsigma \sum_{j \in \mathcal{N}(i)}\left(\mathbf{z}_{j}(h)-\mathbf{z}_{i}(h)\right),
\end{aligned}
$$

which are respectively initialized with $\mathbf{P}_{i}(0)=\mathbf{E}_{i}^{T} \mathbf{E}_{i}$, $\mathbf{z}_{i}(0)=\mathbf{E}_{i}^{T} \mathbf{y}_{i}(k), i \in\{1, \ldots, n\}$. If the step size $\varsigma \in(0,1)$ satisfies the condition ${ }^{1} \varsigma<2 / \lambda_{n}(\mathbf{L})$,

1 Note that the knowledge of the largest eigenvalue of the Laplacian, $\lambda_{n}(\mathbf{L})$, entails the knowledge of the topology of 
cf. [22, Lemma 8.1], then $\lim _{h \rightarrow \infty} \mathbf{P}_{i}(h)=\frac{1}{n} \sum_{i=1}^{n} \mathbf{E}_{i}^{T} \mathbf{E}_{i}$, $\lim _{h \rightarrow \infty} \mathbf{z}_{i}(h)=\frac{1}{n} \sum_{i=1}^{n} \mathbf{E}_{i}^{T} \mathbf{y}_{i}(k)$, and node $i$ asymptotically obtains an estimate $\widehat{\mathbf{x}}^{(i)}$ of vector $\mathbf{x}$ at time $k$ according to:

$$
\widehat{\mathbf{x}}^{(i)}(k)=\lim _{h \rightarrow \infty} \mathbf{P}_{i}^{-1}(h) \mathbf{z}_{i}(h) .
$$

Node $i$ can then compute its local estimate $\widehat{\mathbf{C}}_{\mathrm{R}}^{(i)}$ of $\mathbf{C}_{\mathrm{R}}$ as

$$
\widehat{\mathbf{C}}_{\mathrm{R}}^{(i)}=\frac{1}{\mathrm{~S}} \sum_{k=0}^{\mathrm{S}-1} \widehat{\mathbf{x}}^{(i)}(k)\left(\widehat{\mathbf{x}}^{(i)}(k)\right)^{T},
$$

and eventually obtain an estimate of the Laplacian matrix via equation (12) and the application of $\kappa$-means clustering. Note that the iterates $\mathbf{P}_{i}(h)$ may not be invertible for all values of $h$ : hence, estimate (19) at node $i$ can only be computed once $\mathbf{P}_{i}(h)$ becomes nonsingular. We also observe here that differently from the iterations in (18a), which have to be performed only once, the iterations in $(18 \mathrm{~b})$ must be executed at each time instant $k \in\{0,1, \ldots, \mathrm{S}-1\}$, which is computationally demanding, especially for large networks. In order to reduce the computational load, we can replace (18b) with a dynamic average consensus estimator which tracks the average of all the nodes' inputs $\mathbf{E}_{i}^{T} \mathbf{y}_{i}(k)$ for $k \in\{0,1, \ldots, S-1\}$. Because of its attractive robustness properties, we will make use of the discrete-time version of the PI dynamic average consensus estimator introduced in [23]:

$$
\left\{\begin{aligned}
\mathbf{z}_{i}(k+1) & =(1-\varepsilon \alpha) \mathbf{z}_{i}(k)+\varepsilon \mathrm{K}_{P} \sum_{j \in \mathcal{N}(i)}\left(\mathbf{z}_{j}(k)-\mathbf{z}_{i}(k)\right) \\
& -\varepsilon \mathrm{K}_{I} \sum_{j \in \mathcal{N}(i)}\left(\mathbf{w}_{j}(k)-\mathbf{w}_{i}(k)\right)+\varepsilon \alpha \mathbf{E}_{i}^{T} \mathbf{y}_{i}(k), \\
\mathbf{w}_{i}(k+1) & =\mathbf{w}_{i}(k)+\varepsilon \mathrm{K}_{I} \sum_{j \in \mathcal{N}(i)}\left(\mathbf{z}_{j}(k)-\mathbf{z}_{i}(k)\right),
\end{aligned}\right.
$$

where $\mathbf{z}_{i}$ is node $i$ 's estimate of the average of all the nodes' inputs, $\mathbf{w}_{i}$ is an internal estimator state, $\mathrm{K}_{P}, \mathrm{~K}_{I}>0$ are estimator gains, $\varepsilon>0$ is the step size, and $\alpha>0$ is a parameter governing the rate at which new information replaces old information in the dynamic averaging process (the so-called "forgetting factor"). Each node communicates its estimate $\mathbf{z}_{i}$ and its internalestimator state $\mathbf{w}_{i}$ to its neighbors in the network. Note that differently from a static average consensus protocol, in (20) the inputs continually drive the estimator and each $\mathbf{z}_{i}(k)$ approximately tracks the true average of $\mathbf{E}_{i}^{T} \mathbf{y}_{i}(k)$. By defining $\mathbf{z}=\left[\mathbf{z}_{1}^{T}, \ldots, \mathbf{z}_{n}^{T}\right]^{T} \in \mathbb{R}^{n^{2}}$ and $\mathbf{w}=\left[\mathbf{w}_{1}^{T}, \ldots, \mathbf{w}_{n}^{T}\right]^{T} \in \mathbb{R}^{n^{2}}$, we can rewrite system (20) in compact form as:

$$
\left[\begin{array}{c}
\mathbf{z}(k+1) \\
\mathbf{w}(k+1)
\end{array}\right]=\left(\boldsymbol{\Gamma} \otimes \mathbf{I}_{n}\right)\left[\begin{array}{c}
\mathbf{z}(k) \\
\mathbf{w}(k)
\end{array}\right]+\left[\begin{array}{c}
\varepsilon \alpha \mathbf{I}_{n^{2}} \\
\mathbf{0}
\end{array}\right] \mathbf{E}^{T} \mathbf{y}(k),
$$

the overall network by each node. However, it is well-known that $\lambda_{n}(\mathbf{L}) \leq n[21]$, and the upper bound $n$ can be used by each node to determine $\varsigma$ in (18). where

$$
\boldsymbol{\Gamma}=\left[\begin{array}{cc}
(1-\varepsilon \alpha) \mathbf{I}_{n}-\varepsilon \mathrm{K}_{p} \mathbf{L} & \varepsilon \mathrm{K}_{I} \mathbf{L} \\
-\varepsilon \mathrm{K}_{I} \mathbf{L} & \mathbf{I}_{n}
\end{array}\right]
$$

The proof of the next proposition, which is not available in the literature, is given in the Appendix.

\section{Proposition 3 (Stability of the PI estimator)}

The PI estimator (21) is stable if the step size

$$
\varepsilon<\frac{2 \operatorname{Re}\left(\beta_{i}\right)}{\left|\beta_{i}\right|^{2}}, \quad \forall i \in\{2, \ldots, n\},
$$

where

$$
\beta_{i}=\frac{1}{2}\left[\alpha-\mathrm{K}_{P} \zeta_{i} \pm \sqrt{\left(\alpha-\mathrm{K}_{P} \zeta_{i}\right)^{2}-4 \mathrm{~K}_{I}^{2} \zeta_{i}^{2}}\right]
$$

and $\zeta_{i}, i \in\{2, \ldots, n\}$, is the $i$-th eigenvalue of $-\mathbf{L}$.

Remark 1 As the size of the network $\mathcal{G}$ increases, longer time series are needed to get the same level of reconstruction accuracy (and, in general, to make $\mathbf{P}_{i}(h)$ invertible): in fact, the information takes more time to flow through $\mathcal{G}$ and more communication rounds are necessary. On the other hand, higher noise magnitude may reduce, in general, the number of necessary consensus steps but also increase the power dissipation at each node. Therefore, computational complexity/energy consumption needs to be weighted against reconstruction accuracy in practical applications where available resources are at premium. $\diamond$

\section{Numerical validation}

Numerical simulations have been conducted with five realistic networks in order to compare the performance of the centralized and distributed network reconstruction methods.

- Data and computer networks: A full binary tree is a rooted tree in which every node other than the leaves has two children [21]. Full binary trees are extensively used to organize data in computer systems. In our simulations, we considered the "Full binary tree $\mathcal{T}_{4}$ " which has 4 levels (excluding the root node) and 31 nodes. Hypercube networks [21] have found frequent applications in parallel processing (e.g. in massively-parallel supercomputers). In this study, we chose the "Hypercube $\mathcal{Q}_{4} "$, a regular network with 16 nodes.

- Electric power network: the "IEEE 9-bus" power system consists of four generators (nodes from 1 to 4 ) and five loads (nodes from 5 to 9), see [4].

- Transportation networks: the links of the network "Road Grenoble" correspond to the major arterials in the city of Grenoble in France, and the nodes to 27 road intersections (see Fig. 3(a)). The metro of Lyon, "Metro Lyon", in France, has a total of 4 lines and 42 stations [24]. In this study, only the 6 termini (black) and 4 transfer stations (white) are considered as nodes (see Fig. 3(b)).

Following [1], the network-reconstruction performance was quantified using the success rate of existing and non-existing links (SREL, SRNL, respectively). SREL (SRNL) is defined as the ratio of the number 


\begin{tabular}{|c|c|c|c|c|c|}
\hline Network & \# nodes & \# links & SREL/SRNL Centralized & SREL/SRNL Distributed $(2$ nodes $)$ & $\mathrm{K}_{P}, \mathrm{~K}_{I}, \alpha, \varepsilon, D$ \\
\hline \hline Full binary tree $\mathcal{T}_{4}$ & 31 & 30 & $1.0000 / 1.0000$ & $0.9167 / 0.9722,0.8667 / 0.9733$ & $60,0.05,3,0.005,2500$ \\
\hline Hypercube $\mathcal{Q}_{4}$ & 16 & 32 & $1.0000 / 1.0000$ & $0.9906 / 0.9977,0.9127 / 0.9943$ & $40,0.05,8,0.005,2000$ \\
\hline IEEE 9-bus & 9 & 9 & $1.0000 / 1.0000$ & $0.9000 / 0.9630,0.9667 / 0.9556$ & $50,0.05,1.5,0.005,2000$ \\
\hline Road Grenoble & 27 & 42 & $0.9976 / 0.9997$ & $0.7143 / 0.8829,0.7071 / 0.8903$ & $40,0.05,1.5,0.003,2000$ \\
\hline Metro Lyon & 10 & 10 & $1.0000 / 1.0000$ & $0.7700 / 0.9971,0.8000 / 0.9943$ & $40,0.05,1.5,0.005,2000$ \\
\hline
\end{tabular}

\section{Table 1}

Success rate of existent and non-existent links (SREL/SRNL) with the centralized and distributed reconstruction methods for five different network topologies. The results are the average of 10 trials, each one of 1000 seconds.

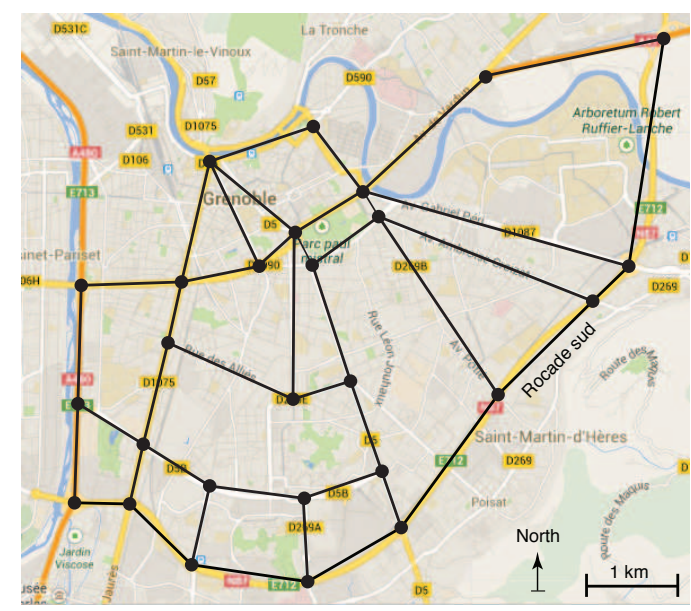

(a)

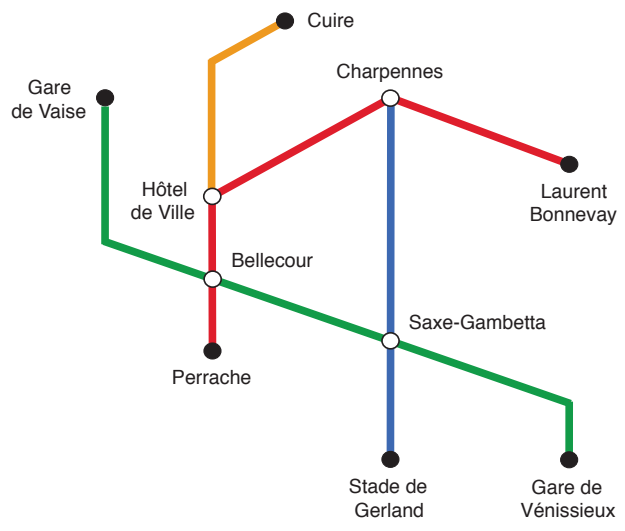

(b)

Fig. 3. Examples: (a) Network of major arterials in the city of Grenoble (image source: Google maps); (b) Simplified plan of the metro of Lyon (www.tcl.fr).

of successfully predicted existent (nonexistent) links to the total number of existent (nonexistent) links. In our tests, the coupling strength $\gamma$ was set to 1 and the noise magnitude $\sigma^{2}=2$. Random initial conditions $\mathbf{x}(0)$, and $\mathbf{z}(0), \mathbf{w}(0)$ were selected for system (10) and the PI estimator (21), respectively. $\kappa$-means clustering was used in all cases for separating the off-diagonal entries of the reconstructed Laplacian matrix, and it produced results more consistent and repeatable than those of the thresholding method in [1]. Table 1 reports the SREL/SRNL obtained as the average of 10 trials of 1000 seconds $(\varsigma=0.1, \mathrm{~S}=10000)$ for the centralized and distributed algorithms, and the value of the parameters used in the numerical tests (last column). In the distributed case, we reported the SREL/SRNL of two adjacent nodes drawn at random in the network: in most of the cases a success rate greater than 0.9 was obtained. In order to illustrate the performance of our dynamic average consensus least-squares algorithm, Fig. 4 shows the time evolution of $x_{j}$ (red) and $\widehat{x}_{j}^{(6)}$ (black), $j \in\{1,2,3,4\}$, obtained via (18a) and (21), for the hypercube network $\mathcal{Q}_{4}$ (note that node 6 is adjacent to node 3 but not to nodes $1,2,4)$. As it is evident from the figure, the estimated state components satisfactorily track the true state.

\section{Conclusions and future work}

In this paper we have presented a fully distributed algorithm for reconstructing the topology of an undirected consensus network. Our approach consists in locally estimating the noise-corrupted network state by using a distributed least-squares algorithm. We proposed a new efficient method for separating the off-diagonal entries of the reconstructed graph Laplacian based on $\kappa$-means clustering, and offered some insight into the trace of the dynamic correlation matrix of the coupled single integrators. Numerical simulations performed on realistic networks have demonstrated the effectiveness of our distributed reconstruction method.

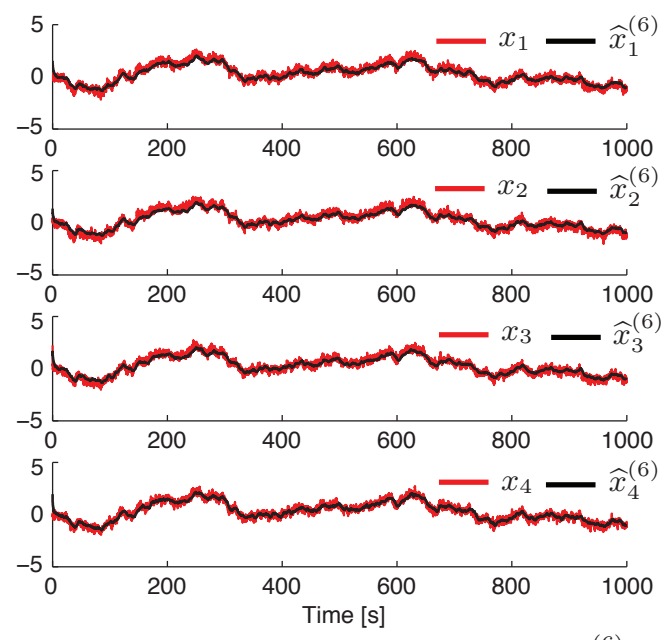

Fig. 4. Time evolution of $x_{j}$ (red) and $\widehat{x}_{j}^{(6)}$ (black), $j \in\{1,2,3,4\}$, for the hypercube network $\mathcal{Q}_{4}$. 
In this paper, we have only dealt with the Boolean structure of a network (i.e. with the existence or nonexistence of links between nodes). In future works, we would like to extend the proposed method to recover the weights of a network, and to estimate the directions of the links in directed graphs. We are also going to explore other methods for computing the sample covariance matrix in equation (14) in a distributed fashion (e.g. by estimating its eigenvectors in a decentralized way), and to relax the assumption of known noise magnitude. Finally, it is worth pointing out that because of its computational complexity, the proposed reconstruction algorithm is not applicable, at present, to networks with hundreds or thousands of nodes. In order to address this problem, we plan to exploit the sparsity of large-scale real-world networks for distributed least-squares estimation, and to leverage the information coming from anchor or ancillary nodes around the network.

\section{Appendix}

Lemma 1 Let $\mathbf{A} \in \mathbb{R}^{n \times n}$ and $\mathbf{B} \in \mathbb{R}^{m \times m}$. Then the eigenvalues of $\mathbf{A} \otimes \mathbf{B}$ are of the form $\lambda_{i}(\mathbf{A}) \lambda_{j}(\mathbf{B}), i \in$ $\{1, \ldots, n\}, j \in\{1, \ldots, m\}$.

Lemma 2 (Schur's formula) Let A, B, C, D $\in$ $\mathbb{R}^{n \times n}$. If $\mathbf{A}$ and $\mathbf{C}$ commute, i.e. $\mathbf{A C}=\mathbf{C A}$, then $\operatorname{det}\left[\begin{array}{ll}\mathbf{A} & \mathbf{B} \\ \mathbf{C} & \mathbf{D}\end{array}\right]=\operatorname{det}(\mathbf{A D}-\mathbf{C B})$.

Proof of Proposition 3: Owing to Lemma 1, to prove the statement we only need to study the eigenvalues of matrix $\boldsymbol{\Gamma}$, which are the roots of $\operatorname{det}\left(\lambda \mathbf{I}_{2 n}-\boldsymbol{\Gamma}\right)=0$. If we apply Lemma 2, we find that

$$
\begin{aligned}
& \operatorname{det}\left(\lambda \mathbf{I}_{2 n}-\boldsymbol{\Gamma}\right)= \\
& \operatorname{det}\left((\lambda-1)\left[(\lambda-1+\varepsilon \alpha) \mathbf{I}_{n}+\varepsilon \mathbf{K}_{P} \mathbf{L}\right]+\varepsilon^{2} \mathbf{K}_{I}^{2} \mathbf{L}^{2}\right) .
\end{aligned}
$$

Note now that $\operatorname{det}\left(\lambda \mathbf{I}_{n}+\mathbf{L}\right)=\prod_{i=1}^{n}\left(\lambda-\zeta_{i}\right)$ where $\zeta_{i}$ is the $i$-th eigenvalue of $-\mathbf{L}$ with $0=\zeta_{1}>\zeta_{2} \geq \ldots \geq \zeta_{n}$. By comparing (24) with this last equation, we see that $\operatorname{det}\left(\lambda \mathbf{I}_{2 n}-\boldsymbol{\Gamma}\right)=\prod_{i=1}^{n}\left(\lambda^{2}-\left(2-\varepsilon \alpha+\varepsilon \mathrm{K}_{P} \zeta_{i}\right) \lambda+1-\right.$ $\left.\varepsilon \alpha+\varepsilon K_{P} \zeta_{i}+\varepsilon^{2} \mathrm{~K}_{I}^{2} \zeta_{i}^{2}\right)$ from which it follows that the eigenvalues of $\boldsymbol{\Gamma}$ can be obtained by solving the equation $\lambda^{2}-\left(2-\varepsilon \alpha+\varepsilon \mathrm{K}_{P} \zeta_{i}\right) \lambda+1-\varepsilon \alpha+\varepsilon \mathrm{K}_{P} \zeta_{i}+\varepsilon^{2} \mathrm{~K}_{I}^{2} \zeta_{i}^{2}=0$. Hence, if $\lambda_{i}^{ \pm}$are the eigenvalues of $\boldsymbol{\Gamma}$ associated with $\zeta_{i}$, we have that

$$
\lambda_{i}^{ \pm}=1-\frac{\varepsilon}{2}\left(\alpha-\mathrm{K}_{P} \zeta_{i} \pm \sqrt{\left(\alpha-\mathrm{K}_{P} \zeta_{i}\right)^{2}-4 \mathrm{~K}_{I}^{2} \zeta_{i}^{2}}\right) .
$$

Note that $\lambda_{1}^{+}=1-\varepsilon \alpha$ and $\lambda_{1}^{-}=1$ since $\zeta_{1}=0$, and that $\lambda_{1}^{-}$is the only eigenvalue of $\boldsymbol{\Gamma}$ having unitary modulus. Let us now define the complex number $\beta_{i}$ as in equation (23) (notice that $\operatorname{Re}\left(\beta_{i}\right) \geq 0, \forall i$ ). Then for system (21) to be stable, we must impose that $\left|1-\varepsilon \beta_{i}\right|<1$, $\forall i \in\{2, \ldots, n\}$, from which condition (22) follows.

\section{References}

[1] J. Ren, W.-X. Wang, B. Li, and Y.-C. Lai. Noise bridges dynamical correlation and topology in coupled oscillator networks. Phys. Rev. Lett., 104(5):058701, 2010.
[2] E.R. van Dam and W.H. Haemers. Developments on spectral characterizations of graphs. Discrete Math., 309(3):576-586, 2009.

[3] M. Nabi-Abdolyousefi and M. Mesbahi. Network identification via node knockout. IEEE Trans. Automat. Contr., 6(12):1926-1932, 2012.

[4] M. Suzuki, N. Takatsuki, J. Imura, and K. Aihara. KnockOut/Down-Based Structure Identification in Networks of Heterogeneous Subsystems. In Proc. 52nd IEEE Conf. Dec. Contr, pages 1343-1348, 2013.

[5] A.Y. Kibangou and C. Commault. Decentralized Laplacian Eigenvalues Estimation and Collaborative Network Topology Identification. In Proc. 3rd IFAC Work. Distrib. Estim. Contr. Net. Syst., pages 7-12, 2012.

[6] D. Hayden, Y. Yuan, and J. Gonçalves. Network Reconstruction from Intrinsic Noise. arXiv: 1310.0375v1 [cs.SY], 2013.

[7] S. Shahrampour and V.M. Preciado. Topology Identification of Directed Dynamical Networks via Power Spectral Analysis. arXiv: 1308.2248v1 [cs.SY], 2013.

[8] D. Materassi, G. Innocenti, L. Giarré, and M. Salapaka. Model identification of a network as compressing sensing. Syst. Contr. Lett., 62(8):664-672, 2013.

[9] F.R. Bach and M.I. Jordan. Learning graphical models for stationary time series. IEEE Trans. Signal Proces., 52(8):2189-2199, 2004.

[10] A. Julius, M. Zavlanos, S. Boyd, and G. Pappas. Genetic network identification using convex programming. IET Syst. Biol., 3(3):155-166, 2009.

[11] J. Gonçalves and S. Warnick. Necessary and sufficient conditions for dynamical structure reconstruction of LTI networks. IEEE Trans. Automat. Contr., 53(7):1670-1674, 2008.

[12] A. Dankers, P.M. Van den Hof, P.S. Heuberger, and $\mathrm{X}$. Bombois. Dynamic network identification using the direct prediction-error method. In Proc. 51st IEEE Conf. Dec. Contr, pages 901-906, 2012.

[13] E. Fioriti, S. Chiesa, and F. Fratichini. Exact Network Reconstruction from Consensus Signals and One Eigenvalue. Int. J. Comput. Net. Comm., 5(5):83-93, 2013.

[14] S.P. Lloyd. Least-squares quantization in PCM. IEEE Trans. Inform. Theory, 28(2):129-137, 1982.

[15] A. Ghosh, S. Boyd, and A. Saberi. Minimizing effective resistance of a graph. SIAM Rev., 50(1):37-66, 2008.

[16] L. Xiao, S. Boyd, and S.-J. Kim. Distributed average consensus with least-mean-square deviation. J. Parallel Distr. Com., 67(1):33-46, 2007.

[17] G.F. Young, L. Scardovi, and N.E. Leonard. Robustness of noisy consensus dynamics with directed communication. In Proc. American Contr. Conf, pages 6312-6317, 2010.

[18] D. Lusseau et al. The bottlenose dolphin community of Doubtful Sound features a large proportion of long-lasting associations. Behav. Ecol. Sociobiol., 54(4):396-405, 2003.

[19] B. Bamieh, M. Jovanović, P. Mitra, and S. Patterson. Coherence in large-scale networks: Dimension-dependent limitations of local feedback. IEEE Trans. Automat. Contr., 57(9):2235-2249, 2012.

[20] F. Blanchini and S. Miani. Set-Theoretic Methods in Control. Birkhäuser, Boston, 2008.

[21] C. Godsil and G. Royle. Algebraic graph theory. Springer, 2001.

[22] M. Mesbahi and M. Egerstedt. Graph Theoretic Methods in Multiagent Networks. Princeton University Press, 2010.

[23] K.M. Lynch, I.B. Schwartz, P. Yang, and R.A. Freeman. Decentralized environmental modeling by mobile sensor networks. IEEE Trans. Robot., 24(3):710-724, 2008.

[24] S. Derrible. Network centrality of metro systems. PloS one, 7(7):e40575, 2012. 